\title{
Schwerpunkt: 30 Jahre Deutsche Einheit
}

\section{Arno von Schuckmann}

Online publiziert: 4. August 2020

(C) Der/die Autor(en) 2020

Als vor 31 Jahren die Mauer fiel und sich politische Wege hin zur Deutschen Einheit offenbarten, wurde von vielen euphorisch eine erfolgreiche gemeinsame Zukunft der beiden Länder gezeichnet. Insbesondere der Glaube, dass das kapitalistische Wirtschaftssystem, welches sich gegen den planwirtschaftlichen Sozialismus behauptet hatte, auch eine rasche Angleichung der Lebens- und Wirtschaftsbedingungen in Ost- und Westdeutschland herbeiführen würde, überdeckte zu Beginn der Deutschen Einheit vor 30 Jahren die strukturellen Probleme Ostdeutschlands.

Neben den erheblichen Produktivitätsdefiziten der ostdeutschen Wirtschaft, die schon sehr früh nach der Einheit zutage traten, offenbarten sich zudem sukzessive die divergierenden Sichtweisen auf das demokratische System des vereinten Deutschlands. Diese zeigten sich unter anderem im politischen Protest an der Wahlurne. So gelang es lange Zeit der SED-Nachfolgepartei PDS ${ }^{1}$ in Ostdeutschland auf Volksparteienniveau bei den Landtagswahlen sowie Bundestagswahlen abzuschneiden. Mit zunehmender Systemkonformität der Partei und beschleunigt durch die tiefgreifenden Krisen der 2010er Jahre suchte sich der politische Protest deutschlandweit - aber insbesondere in Ostdeutschland - einen neuen Weg.

Spätestens mit dem starken Abschneiden der rechtspopulistischen AfD in den ostdeutschen Bundesländern bei der Bundestagswahl 2017 wurde evident, dass die Bundesrepublik Deutschland auch heute noch ein teils politisch gespaltenes Land ist. Die Erklärungsmuster für diese Trennung sind vielfältig und reichen von den weiterhin sehr ungleichen Lebensverhältnissen in Ost und West - man denke nur

1 Seit 2007 Die Linke.

A. von Schuckmann $(\bowtie)$

Institut für Politikwissenschaft, NRW School of Governance, Universität Duisburg-Essen,

Lotharstraße 53, 47057 Duisburg, Deutschland

E-Mail: arno.vonschuckmann@uni-due.de 
an die unterschiedlichen Löhne oder an sogenannte abgehängte Regionen - bis hin zur fehlenden Sozialisierung in einem demokratischen Staat. Sicher haben all diese Erklärungsversuche ihre Berechtigung, doch greifen sie häufig zu kurz. Aus diesem Grunde ist es auch für die Politikwissenschaft besonders wichtig, das diesjährige Jubiläum zu nutzen, um einen Blick auf das vereinte Deutschland zu werfen.

Beginnen wird Karl-Rudolf Korte mit einer prägnanten Nachzeichnung der Geschehnisse der Jahre 1989/90 sowie dem Politikmanagement der Regierung Kohl. Anschließend widmet sich Marcus Böick der Wahrnehmung und Bedeutung der Treuhandanstalt und versucht so einen möglichen Brückenschlag zwischen Politikwissenschaft und Zeitgeschichte darzulegen, bevor sich Susanne und Gert Pickel der Frage widmen, ob es in Deutschland eine gemeinsame demokratische politische Kultur gibt. Abschließend geht Everhard Holtmann der Frage nach, wie sich die Ost-West-Differenz überwinden lässt.

Funding Open Access funding provided by Projekt DEAL.

Open Access Dieser Artikel wird unter der Creative Commons Namensnennung 4.0 International Lizenz veröffentlicht, welche die Nutzung, Vervielfältigung, Bearbeitung, Verbreitung und Wiedergabe in jeglichem Medium und Format erlaubt, sofern Sie den/die ursprünglichen Autor(en) und die Quelle ordnungsgemäß nennen, einen Link zur Creative Commons Lizenz beifügen und angeben, ob Änderungen vorgenommen wurden.

Die in diesem Artikel enthaltenen Bilder und sonstiges Drittmaterial unterliegen ebenfalls der genannten Creative Commons Lizenz, sofern sich aus der Abbildungslegende nichts anderes ergibt. Sofern das betreffende Material nicht unter der genannten Creative Commons Lizenz steht und die betreffende Handlung nicht nach gesetzlichen Vorschriften erlaubt ist, ist für die oben aufgeführten Weiterverwendungen des Materials die Einwilligung des jeweiligen Rechteinhabers einzuholen.

Weitere Details zur Lizenz entnehmen Sie bitte der Lizenzinformation auf http://creativecommons.org/ licenses/by/4.0/deed.de. 